\title{
ARTIGOS
}

\section{Manejo da requeima do tomateiro industrial empregando sistema de previsão}

\author{
Henrique da Silva Silveira Duarte ${ }^{1}$, Laércio Zambolim ${ }^{1}$, Waldir Cintra de Jesus Junior ${ }^{2}$
}

\begin{abstract}
${ }^{1}$ Departamento de Fitopatologia, Universidade Federal de Viçosa, Av.: P.H. Rolfs, s/n, CEP 36.570-000, Viçosa, MG.,e-mail: zambolim@ufv.br. ${ }^{2}$ Departamento de Produção Vegetal, Universidade Federal do Espírito Santo, Alto Universitário, s/n, CEP 29.500-000, Alegre, ES. Autor para correspondência: Laércio Zambolim

Data da chegada: 27/03/2006. Aceito para publicação em: 30/4/2007.
\end{abstract}

\section{RESUMO}

Duarte, H. da S. S.; Zambolim, L; Jesus Junior, W. C. de. Manejo da requeima do tomateiro industrial empregando sistema de previsão. Summa Phytopathologica, v.33, n.4, p.328-334, 2007.

Três experimentos foram conduzidos utilizando-se o híbrido de tomateiro industrial H $7155 \mathrm{~N}$, em 2003 e 2004 visando adequar um sistema de previsão modificado para o uso de fungicidas no manejo da requeima causada por Phytophthora infestans. Os experimentos foram conduzidos num delineamento em blocos ao acaso com dez tratamentos e três repetições. Os fungicidas sistêmicos foram aplicados quando o valor de severidade da doença (VSD) alcançou oito, dez ou doze pontos. A alternância dos fungicidas sistêmicos com o metiram ocorreu na semana em que não foram alcançados os VSD's. O tratamento calendário de pulverização para o controle da requeima consistiu da aplicação semanal de fungicidas após o aparecimento dos primeiros sintomas e aqueles definidos pelo sistema de previsão foram baseados nos VSD acumulados durante o experimento. A avaliação da requeima foi feita semanalmente com auxílio de uma escala descritiva de severidade e uma chave diagramática. A eficiência dos tratamentos foi comparada calculando-se a área abaixo da curva do progresso da doença, a taxa de progresso da doença $(r)$ e produtividade de tomate. Os valores de $r$ do melhor tratamento (dimetomorfe misturado com clorotalonil $(\mathrm{VSD}=10)$ alternado com metiram variaram de 0,03 a 0,07 , enquanto para o tratamento calendário semanal foi de 0,05 a 0,09 e para testemunha de 0,24 a 0,39 . Dimetomorfe + clorotalonil $(\mathrm{VSD}=10)$ alternado com metiram produziu em média 15,7 toneladas a mais de tomate, comparado ao tratamento calendário semanal de fungicidas. Dimetomorfe ou metalaxil-M misturado com clorotalonil (VSD = 10) baseado no sistema de previsão sem a alternância com metiram diferiu do calendário semanal baseado na severidade da doença e na produção de tomate. Os tratamentos piraclostrobina misturado com metiram $(\mathrm{VSD}=8 ; \mathrm{VSD}=10)$ alternado com metiram; dimetomorfe misturado com mancozeb (VSD $=8$; VSD $=$ 10) alternado com metiram proporcionaram controle intermediário da requeima. O sistema de previsão baseado no uso de dimetomorfe misturado com clorotalonil alternado com metiram (VSD = 10) permitiu redução de uma aplicação da mistura dimetomorfe misturado com clorotalonil pulverização em dois dos três experimentos, comparados ao tratamento calendário semanal de aplicação de fungicidas, sem afetar a produção.

Palavras-chave adicionais: Phytophthora infestans, Lycopersicon esculentum Mill, manejo integrado de doença

\section{ABSTRACT}

Duarte, H. da S. S.; Zambolim, L; Jesus Junior, W. C. de. Management of industrial tomato late blight using prediction system. Summa Phytopathologica, v.33, n.4, p.328-334, 2007.

Three experiments were carried out using an industrial tomato hybrid H $7155 \mathrm{~N}$ crop, in 2003 and 2004 seasons to fit a modified prediction system, which allows the criterious use of fungicides for the control of late blight caused by Phytophthora infestans. Trials were conducted in a randomized complete block design with ten treatments and three replications. The formulated systemic fungicides were applied when the disease severity values (DSV) reached eight, ten or twelve points. On weeks where DSV's values were lower than eight, ten or twelve, metiram was applied. The traditional late blight control consisted of weekly application of fungicides after the first symptoms disease, and those defined by the prediction system were carried out based on the DSV's accumulated throughout the experiment. Late blight severity was assessed weekly by using a descriptive key and diagrammatic scale. The efficiency of the control treatments was compared by the area under the disease progress curve, disease progress rate $(r)$ and tomato yield. The $r$ values of the best treatment (dimethomorph + chlorothalonil, DSV $=$
10 , alternated with metiram), weekly application calendar fungicides and the check treatment varied from 0.03 to $0.07,0.05$ to 0.09 and 0.24 to 0.39 , respectively. Dimethomorph + chlorothalonil DSV $=10$ alternated with metiram was the most efficient and yielded more than 15,7 ton of tomato in average, above the weekly spraying calendar fungicides. Dimethomorph or metalaxyl-M mixtured with chlorothalonil $(\mathrm{DSV}=10)$ based on the prediction scheme without alternation with metiram differed from the weekly spraying calendar fungicide based on disease severity and tomato yield. The treatments pyraclostrobim + metiram $(\mathrm{DSV}=8 ; \mathrm{DSV}=10)$ alternated with metiram; dimethomorph + mancozeb $(\mathrm{DSV}=8$; $\mathrm{DSV}=10$ ) alternated with metiram gave intermediate control of late blight and tomato yield. The prediction scheme that used dimethomorph + chlorothalonil alternated with metiram (DSV $=$ 10) allowed the reduction in one spray in two trials of the mixture dimethomorph + chlorothalonil compared to the weekly spraying calendar fungicide without any yield loss. 
O tomateiro (Lycopersicon sculentum Mill) é considerado uma das culturas mais expressivas no cenário agrícola mundial devido sua importância para o comércio "in natura" e indústria de extratos. Atualmente a tomaticultura brasileira encontra-se presente em todas as regiões do país, sendo as regiões centro-oeste e sudeste os principais centros de produção $(2,21)$.

A produção de tomate no Brasil é limitada por vários fatores, sendo as doenças um dos principais problemas. Dentre as doenças que atacam o tomateiro não só no Brasil, mas em todo o mundo, a requeima causada por Phytophthora infestans (Mont.) de Bary, é uma das doenças mais destrutivas dessa olerícola, podendo comprometer todo o campo de produção em poucos dias $(13,22)$ sendo considerada uma das doenças de plantas mais devastadoras de toda humanidade (26).

A requeima ocorre em praticamente todos os locais onde o tomate é cultivado, sendo mais severa em períodos frios e úmidos. É favorecida por temperaturas moderadas, $12-20^{\circ} \mathrm{C}$ e molhamento foliar superior a 10 horas, podendo ocorrer em regiões de clima quente, desde que as noites sejam frias $(6,26)$. Em temperaturas acima de $30^{\circ} \mathrm{C}$, a requeima dificilmente ocorre, mas o patógeno permanece vivo e pode provocar danos assim que as condições climáticas forem favoráveis (13). A requeima pode afetar severamente folhas, hastes, frutos e pecíolos apresentando um aspecto semelhante à queima $(11,12)$.

O tomate tem sido cultivado em épocas favoráveis à requeima e o problema agrava-se com o uso de cultivares suscetíveis, as quais são preferidas em função da alta produtividade e das boas características agronômicas. Com isso após o transplantio das mudas ao campo são iniciadas as pulverizações e seguem até o final do ciclo da cultura, em intervalos de três a cinco dias (18). Há relatos de realização de até 30 pulverizações por ciclo da cultura (14). As pulverizações são feitas sem a observação de nenhum critério de ocorrência do patógeno ou de condições ambientais favoráveis à doença (25). Desta maneira, o custo de produção torna-se onerado além de causar outros problemas, tais como: intoxicação de aplicadores, contaminação do meio ambiente pela deriva, lixiviação dos excessos de produtos pulverizados, resíduos de fungicidas em frutos e outros órgãos vegetais, surgimento de isolados resistentes do patógeno, etc. Todas essas implicações tem sido motivo de preocupação e têm levado a procura crescente por práticas e estratégias racionais de manejo da requeima, além da busca por fungicidas de menor custo e toxicidade $(20,28)$.

Várias medidas têm sido estudadas, visando tornar a cultura do tomateiro mais rentável e otimizar o uso de produtos químicos para o controle da requeima. Dentre essas medidas, o emprego de sistemas de previsão de doenças tem se destacado como alternativa para otimizar o uso de produtos químicos, isto porque o manejo leva em consideração a biologia do patógeno e suas interações com as variáveis climáticas.

Dentre os resultados obtidos com aplicação de modelos de previsão de doenças destacam-se a possibilidade de maior lucro ao produtor, o decréscimo do risco de ocorrência de epidemias, a redução da poluição ambiental, a determinação do momento adequado para aplicação de fungicidas e a redução do número de pulverizações (1).

A meta dos sistemas de previsão é determinar o momento adequado de aplicação de fungicidas em função do monitoramento de condições favoráveis ao desenvolvimento da doença, aumentando a eficiência das aplicações $(7,19)$. Por outro lado, os calendários de pulverizações adotados pelos produtores não levam em consideração a influência de fatores climáticos no progresso da requeima e não discriminam épocas favoráveis à sua ocorrência. Isto na maioria dos casos resulta em uso excessivo de produtos químicos, maior custo de produção e maiores prejuízos ao homem e ao meio ambiente.

Este estudo teve como objetivo avaliar a viabilidade do uso do sistema de previsão de Wallin (1962) (27) no controle da requeima do tomateiro industrial, de modo a definir intervalos adequados de aplicação de fungicidas.

\section{MATERIAL E MÉTODOS}

\section{Condução do experimento}

Três experimentos foram conduzidos no Departamento de Fitopatologia da Universidade Federal de Viçosa utilizando-se o híbrido de tomate industrial $\mathrm{H} 7155 \mathrm{~N}$, suscetível à requeima. Os experimentos foram conduzidos empregando-se delineamento em blocos casualizados, com dez tratamentos e três repetições. Os experimentos 1, 2 e 3 foram conduzidos respectivamente nos períodos, respectivamente: de 13 de julho a 12 de outubro de 2003 , de 07 de maio a 13 de agosto de 2004 e de 17 de setembro a 12 de dezembro de 2004. As mudas foram transplantadas para o campo aos 25 dias após a semeadura, quando as plantas apresentaram cinco pares de folhas definitivas usando espaçamento de $0,30 \mathrm{~m}$ entre plantas e 1,0 m entre fileiras. Cada parcela foi composta de 40 plantas divididas em quatro fileiras, sendo duas fileiras laterais deixadas como bordadura. Dentro das fileiras centrais uma planta do final de cada linha foi deixada como bordadura, resultando em 16 plantas úteis na parte central da parcela.

Os tratamentos consistiram da aplicação de fungicidas sistêmicos e protetores de acordo com o sistema de previsão e o calendário semanal de pulverização para o controle da requeima (Tabelas 1 e 2). Foram utilizados os seguintes fungicidas com as respectivas doses em gramas de ingrediente ativo por hectare $(\mathrm{g}$ i.a./ha): Dimetomorfe + clorotalonil $(239,7+1.198,5$ g i.a./ha), piraclostrobina + metiram $(150+1650 \mathrm{~g}$ i.a./ha $)$, metalaxil-M + mancozeb $(100+1600$ g i.a./ha), dimetomorfe + mancozeb $(180+$ $1200 \mathrm{~g}$ i.a./ha) e metalaxil-M + clorotalonil $(101,3+1012,5 \mathrm{~g}$ i.a./ ha) e os protetores metiram (2100 g i.a./ha), mancozeb ( $2400 \mathrm{~g}$ i.a./ha) e clorotalonil (1500 g i.a./ha). O tratamento calendário de pulverização para o controle da requeima consistiu da aplicação semanal de fungicidas após o aparecimento dos primeiros sintomas e aqueles definidos pelo sistema de previsão foram baseados nos VSD acumulados durante o experimento. As pulverizações foram realizadas com pulverizador costal manual de 20 litros de capacidade com bico de pulverização do tipo cone vazio. O volume de calda fungicida utilizado foi de $1000 \mathrm{l} / \mathrm{ha}$.

Houve a alteração dos tratamentos do experimento 3 visando avaliar a eficiência do fungicida metalaxil-M + clorotalonil, tanto no sistema de previsão quanto no sistema de calendário semanal.

\section{Coleta dos dados meteorológicos}

$\mathrm{Na}$ área experimental foi instalado um abrigo de madeira contendo o equipamento COLPAN 40, dotado de sensores de molhamento e temperatura, além de uma bateria de $6 \mathrm{v}$ e uma placa solar, que foi responsável por manter carregada a bateria do equipamento. Os respectivos sensores foram fixados a um suporte de madeira, na altura do terço médio entre as folhas das plantas, verticalmente.

Durante o período de molhamento foliar, o equipamento registrava a temperatura a cada cinco minutos e assim que o sensor secava as 
Tabela 1. Esquemas de pulverização empregados nos diferentes tratamentos para o controle da requeima do tomateiro nos experimentos 1 e 2 .

\begin{tabular}{|c|c|}
\hline Tratamentos & Esquema de pulverização \\
\hline $\begin{array}{l}\text { 1- Testemunha } \\
\text { 2- Dimetomorfe + clorotalonil } \\
\text { 3- Dimetomorfe + clorothalonil } \\
\text { 4- Dimetomorfe + mancozeb } \\
\text { 5- Dimetomorfe + mancozeb } \\
\text { 6- Piraclostrobina + metiram } \\
\text { 7- Piraclostrobina + metiram } \\
\text { 8- Calendário semanal } \\
\text { 9- Dimetomorfe + mancozeb } \\
\text { 10- Dimetomorfe + clorotalonil }\end{array}$ & $\begin{array}{l}\text { Sem pulverização } \\
\mathrm{VSD}=10 \\
\mathrm{VSD}=12 \\
\mathrm{VSD}=8 \\
\mathrm{VSD}=10 \\
\mathrm{VSD}=8 \\
\mathrm{VSD}=10 \\
\mathrm{Alternados} \text { a cada sete dias } \\
\mathrm{VSD}=8 \\
\mathrm{VSD}=10\end{array}$ \\
\hline
\end{tabular}

\footnotetext{
${ }^{1}$ Nas semanas em que não foi prevista nenhuma pulverização pelo sistema de previsão, estes tratamentos receberam aplicação de um fungicida protetor (Metiram).

${ }_{13}^{12}$ Dimetomorfe + clorotalonil - Mancozeb - Metalaxil-M + mancozeb - Clorotalonil

${ }^{3}$ Pulverizado somente de acordo com o sistema de previsão, nas semanas em que não foi previsto nenhuma pulverização pelo esquema de previsão, estes tratamentos não receberam aplicação de um fungicida protetor (Metiram).

VSD - valor de severidade acumulado da doença.
}

Tabela 2. Esquemas de pulverização empregados nos diferentes tratamentos para o controle da requeima do tomateiro no experimento 3 .

\begin{tabular}{|c|c|}
\hline Tratamentos & Esquema de pulverização \\
\hline $\begin{array}{l}\text { 1-Testemunha } \\
\text { 2- Dimetomorfe + clorotalonil } \\
\text { 3- Dimetomorfe + clorotalonil } \\
\text { 4- Metalaxil-M + clorotalonil } \\
\text { 5- Metalaxil-M + clorotalonil } \\
\text { 6- Dimetomorfe + mancozeb } \\
\text { 7- Piraclostrobina + metiram } \\
\text { 8- Calendário semanal } \\
\text { 9- Metalaxil-M + clorotalonil } \\
\text { 10- Dimetomorfe + clorotalonil }\end{array}$ & $\begin{array}{l}\text { Sem pulverização } \\
V S D=10 \\
V S D=12 \\
V S D=10 \\
V S D=12 \\
V S D=8 \\
V S D=8 \\
\text { Alternados a cada sete dias } \\
\text { VSD }=10 \\
\text { VSD }=10\end{array}$ \\
\hline
\end{tabular}

\footnotetext{
${ }^{11}$ Nas semanas em que não foi prevista nenhuma pulverização pelo sistema de previsão, estes tratamentos receberam aplicação de um fungicida protetor (Metiram).

${ }^{2}$ Dimetomorfe + clorotalonil - Mancozeb - Metalaxil-M + clorotalonil - Clorotalonil

${ }^{3}$ Pulverizado somente de acordo com o sistema de previsão, nas semanas em que não foi previsto nenhuma pulverização pelo esquema de previsão, estes tratamentos não receberam aplicação de um fungicida protetor (Metiram).

${ }^{14}$ VSD - valor de severidade acumulado da doença.
}

informações até então registradas eram processadas e comparadas automaticamente com a tabela de Wallin modificada (Tabela 3), gerando como resultado o valor diário de severidade da doença (VSD) no visor de cristal líquido do equipamento. O VSD foi atribuído arbitrariamente à relação específica entre a duração do período de molhamento foliar e da temperatura média durante o molhamento, variando de 0 a 4 conforme a menor ou maior favorabilidade à doença, respectivamente. Assim, após o plantio das mudas de tomate para o campo foram calculados os VSD's diários, sendo acumulados até atingirem a faixa de VSD previamente fixada para cada tratamento (Tabelas 1 e 2). A partir da primeira pulverização, aguardava-se novo acúmulo de dados até atingir novamente os VSD's estabelecidos para cada tratamento. Em caso de ocorrência dez dias consecutivos desfavoráveis à doença (valores de VSD's iguais à zero), os VSD's eram zerados e nova contagem era iniciada.

Adicionalmente foi instalada uma estação meteorológica (Davis Vantage Pro) ao lado dos experimentos na qual foram obtidos os valores de temperaturas máxima, média e mínima e horas de molhamento foliar diários.

\section{Quantificação do progresso da requeima e da produção}

As avaliações da percentagem de área foliar lesionada foram realizadas semanalmente a partir do aparecimento dos primeiros sintomas até a colheita, em 16 plantas marcadas por parcela, empregando-se escala diagramática e chave descritiva adaptada de James (1971) (8).

As colheitas foram feitas quando os frutos atingiram o estádio verde-maduro ou coloração verde-pálida (15), sendo nesse momento

Tabela 3. Cálculo dos valores de severidade da doença (VSD), em função da duração do período de molhamento foliar e da temperatura média.

\begin{tabular}{cccccc}
\hline $\begin{array}{c}\text { Temperatura } \\
\text { Média }\left({ }^{\circ} \mathrm{C}\right)\end{array}$ & Duração do Período de Molhamento Foliar (horas) \\
\hline $7,2-11,6$ & $0-15$ & $16-18$ & $19-21$ & $22-24$ & $24+$ \\
$11,7-15,0$ & $0-12$ & $13-15$ & $16-18$ & $19-21$ & $22+$ \\
$15,1-26,6$ & $0-9$ & $10-12$ & $13-15$ & $16-18$ & $19+$ \\
VSD $^{11}$ & 0 & 1 & 2 & 3 & 4 \\
\hline
\end{tabular}

Adaptado de Wallin (1962).

Valores de Severidade da Doença variando de zero (condições de ambiente desfavorável ao desenvolvimento da requeima) a quatro (condições de maior favorabilidade).

efetuada a pesagem dos frutos totais por parcela.

\section{Análise dos dados}

Os valores médios de severidade cada tratamento foram utilizados para calcular a área abaixo da curva de progresso da doença (AACPD) e a taxa de progresso da doença $(r)$. Os valores de AACPD calculados pelo programa AVACPD (24), foram divididos pelo número de dias de duração da epidemia com o intuito de padronizar o referido valor. Os valores de $r$ das curvas de severidade da requeima foram estimadas pelo parâmetro $b$ da equação de regressão, obtidas a partir do modelo logístico, que permitiu melhor ajuste dos dados para os tratamentos dos três experimentos. Os valores de AACPD, $r$ e os dados de produtividade de cada tratamento foram comparados pelo teste Tukey, a $5 \%$ de probabilidade, utilizando-se o SAEG (Sistema de Análise Estatística e Genética, Universidade Federal de Viçosa).

\section{RESULTADOS E DISCUSSÃO}

Os dados climáticos, temperatura e horas de molhamento foliar para os três experimentos estão representados na Figura 1. No experimento 1 a temperatura média diária variou de 13,8 a $26,3{ }^{\circ} \mathrm{C}$, a média de horas de molhamento foliar foi de 9,5 horas e a variação foi de 4 a 16 horas de molhamento foliar diário (Figura 1A). As condições climáticas neste experimento foram pouco favoráveis à requeima devido à ocorrência de apenas 19 dias em que a temperatura foi favorável a doença e a média de horas de molhamento foliar foi superior a 10 horas. No experimento 2 a temperatura média diária variou de 9,2 a $20,8^{\circ} \mathrm{C}$, a média de horas de molhamento foliar foi de 13,5 horas e a variação foi de 5,5 a 20 horas de molhamento foliar diário (Figura 1B). As condições climáticas neste experimento foram muito favoráveis a requeima devido à ocorrência de 66 dias com temperatura e duração do molhamento foliar favoráveis à doença. No experimento 3 , a temperatura média diária variou de 16,1 a $25,6{ }^{\circ} \mathrm{C}$, a média de horas de molhamento foliar foi de 12,0 horas e a variação foi de 5 a 24 horas de molhamento foliar diário (Figura 1C). As condições climáticas neste experimento foram pouco favoráveis à requeima devido à ocorrência de apenas 16 dias em que a temperatura foi favorável e a doença e a média de horas de 
molhamento foliar foi superior a 10 horas.

No experimento 1, o tratamento testemunha apresentou $100 \%$ de severidade aos 84 dias após o transplantio. Os tratamentos 2 e 3 , baseados no sistema de previsão e o tratamento 8 baseado no calendário de aplicação semanal apresentaram severidade final 2,0, 3,0 e $2,6 \%$, respectivamente. Os tratamentos 9 e 10 que consistiram
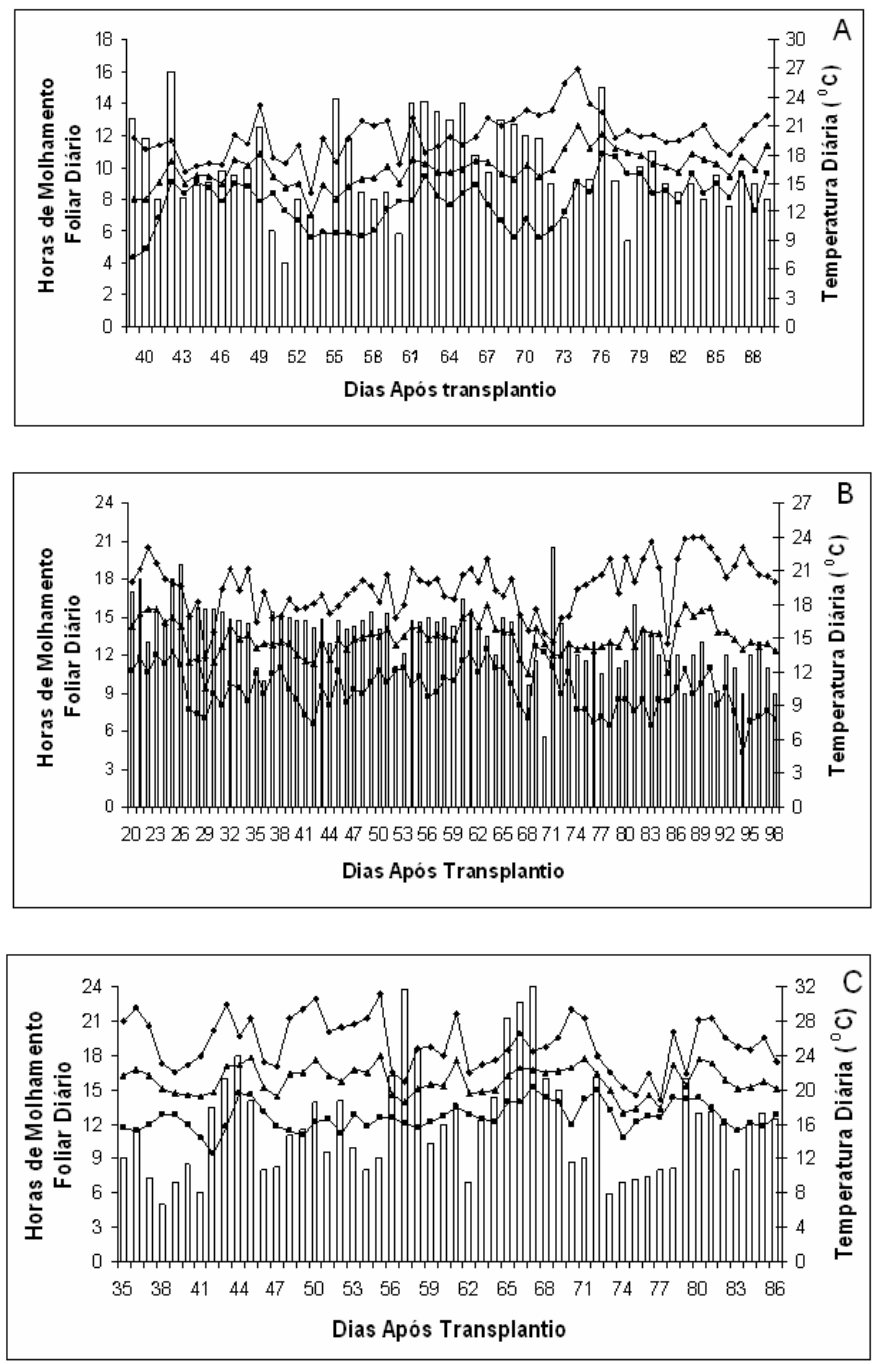

Figura 1. Temperatura máxima, média e mínima (linhas) e horas de molhamento foliar (colunas) durante a epidemia para os experimentos 1 (A), 2 (B) e 3 (C).

apenas na aplicação de produto sistêmico de acordo com o sistema de previsão apresentaram severidade final de 25,0 e $24,7 \%$, respectivamente. Os tratamentos $4,5,6$ e 7 baseados no sistema de previsão apresentaram severidade final de 8,0, 9,5, 6,6 e 6,5\%, respectivamente. O controle mais eficiente da requeima foi obtido pelos tratamentos 2, 3 e 8 devido os quais apresentaram menores valores de Áreas Abaixo da Curva de Progresso da Doença (AACPD), menores taxas de progresso da doença $(r)$ e produtividades superiores (Tabela 4). Os tratamentos 4, 5, 6 e 7 apresentaram controle intermediário da requeima. Os tratamentos 9 e 10 apresentaram baixa eficiência no controle da requeima. Os tratamentos 2 e 3 baseados no sistema de previsão foram eficientes no controle da requeima, e apresentaram menor número de pulverizações com fungicida sistêmico, em uma e duas aplicações, respectivamente, comparados ao tratamento 8 baseado no calendário de aplicação semanal, sem que houvesse perda significativa na produtividade (Tabela 4).

No experimento 2, o tratamento testemunha apresentou $100 \%$ de severidade aos 59 dias após o transplantio. O tratamento 2 baseou-se no sistema de previsão e apresentou um severidade final de 3,33\%. Os tratamentos 9 e 10 que consistiram apenas na aplicação de produto sistêmico de acordo com o sistema de previsão apresentaram severidade final de 58,3 e $30,0 \%$, respectivamente. Os tratamentos 3, 4, 5, 6 e 7, baseados no sistema de previsão e o tratamento 8 , baseado no calendário de aplicação semanal apresentaram severidade final de $9,1,15,0,29,1,13,3,15,8$ e 11,1\%, respectivamente. $\mathrm{O}$ controle mais eficiente da requeima foi obtido através do tratamento 2 apresentando menor valor de AACPD e produtividade significativamente superior (Tabela 4). Os tratamentos 9 e 10 foram pouco eficientes no controle da requeima. Os tratamentos 3, 4, 5, 6, 7 e 8 apresentaram controle intermediário da doença. O tratamento 2 , baseado no sistema de previsão foi o mais eficiente no controle da requeima, porém apresentou aumento no número de pulverizações com fungicida sistêmico em duas aplicações, compensado pela produtividade significativamente superior comparada aos demais tratamentos (Tabela 4).

No experimento 3, o tratamento testemunha apresentou $100 \%$ de severidade aos 86 dias após o transplantio. Os tratamentos 2 e 3 , baseados no sistema de previsão e o tratamento 8 , baseado no calendário de aplicação semanal apresentaram severidade final de $2,6,4,3$ e 5,5\%, respectivamente. Os tratamentos 9 e 10 que consistiram apenas na aplicação de produto sistêmico de acordo com o sistema de previsão apresentaram severidade final de 50,0 e $31,6 \%$, respectivamente. Os tratamentos $4,5,6$ e 7, baseados no sistema de previsão apresentaram severidade final de 12,3, 14,1, 13,3 e $10,8 \%$, respectivamente. O controle mais eficiente da requeima foi obtido pelos tratamentos 2,3 e 8 apresentando menores valores de $r$ e produtividades superiores (Tabela 5). Os tratamentos 4, 5, 6 e 7 apresentaram controle intermediário da requeima. Os tratamentos 9 e 10 apresentaram baixa eficiência no controle da requeima, comparados aos demais tratamentos. Os tratamentos $2 \mathrm{e}$ 3 , baseados no sistema de previsão, foram eficientes no controle da requeima, e apresentaram redução no número de pulverizações com fungicida sistêmico em uma e duas aplicações, respectivamente, comparados ao tratamento 8, baseado no calendário de aplicação semanal, sem que houvesse perda significativa na produtividade (Tabela 5).

Dimetomorfe + clorotalonil $(\mathrm{VSD}=10)$ alternado com metiram produziu em média para os três experimentos 15,7 toneladas a mais de tomate, comparado ao tratamento calendário semanal de fungicidas. Os resultados obtidos nestes experimentos são coerentes com os dados na literatura no que diz respeito às condições climáticas favorecendo o desenvolvimento da requeima. As epidemias de requeima são mais rápidas e severas quando as temperaturas estão na faixa de $12-16^{\circ} \mathrm{C}$ durante a noite. Essa faixa de temperatura favorece a germinação indireta dos esporângios, aumentando o seu poder infectivo e, consequentemente, o desenvolvimento da doença. (17). À medida que a temperatura se distancia das condições ótimas é necessário um período de molhamento foliar mais prolongado para o desenvolvimento da requeima. $(23,29)$.

Neste estudo verificou-se que quando as condições climáticas foram pouco favoráveis a requeima, observadas nos experimentos 1 e 3 , foi possível reduzir o número de pulverizações com fungicidas 
Tabela 4. Número de pulverizações com fungicidas sistêmicos e protetores, área abaixo da curva de progresso da doença (AACPD), taxa de progresso da doença $(r)$ e produtividade influenciada pela requeima do tomateiro nos experimentos 1 e 2 .

\begin{tabular}{|c|c|c|c|c|c|c|c|c|c|c|c|c|c|c|c|c|}
\hline \multirow{3}{*}{ TRAT ${ }^{\}$} & \multicolumn{8}{|c|}{ EXPERIMENTO 1} & \multicolumn{8}{|c|}{ EXPERIMENTO 2} \\
\hline & \multicolumn{2}{|c|}{$\begin{array}{c}\mathrm{N}^{0} \mathrm{de} \\
\text { pulverizações }\end{array}$} & \multirow{2}{*}{\multicolumn{2}{|c|}{$r$}} & \multirow{2}{*}{\multicolumn{2}{|c|}{ AACPD }} & \multirow{2}{*}{\multicolumn{2}{|c|}{$\begin{array}{c}\text { Produtividade } \\
(\mathrm{Kg} / \mathrm{ha})\end{array}$}} & \multicolumn{2}{|c|}{$\begin{array}{c}\mathrm{N}^{0} \text { de } \\
\text { pulverizações }\end{array}$} & \multirow{2}{*}{\multicolumn{2}{|c|}{$r$}} & \multirow{2}{*}{\multicolumn{2}{|c|}{ AACPD }} & \multirow{2}{*}{\multicolumn{2}{|c|}{$\begin{array}{l}\text { Produtividade } \\
(\mathrm{Kg} / \mathrm{ha})\end{array}$}} \\
\hline & $\begin{array}{l}\mathrm{S} \\
-\end{array}$ & $\begin{array}{l}\mathbf{P} \\
-\end{array}$ & & & & & & & S & $\begin{array}{l}\mathbf{P} \\
-\end{array}$ & & & & & & \\
\hline 2 & 4 & 5 & 0,07 & $\mathrm{c}$ & 0,80 & $\mathrm{f}$ & $48.148,0$ & a & 8 & 5 & 0,03 & $\mathrm{c}$ & 1,36 & f & $52.518,5$ & a \\
\hline 3 & 3 & 6 & 0,09 & bc & 1,09 & $\mathrm{f}$ & $46.381,2$ & a & 6 & 6 & 0,05 & $\mathrm{c}$ & 4,02 & ef & $28.851,8$ & $\mathrm{~b}$ \\
\hline 4 & 5 & 4 & 0,12 & $\mathrm{~b}$ & 3,30 & de & $30.413,0$ & $\mathrm{~b}$ & 10 & 3 & 0,05 & $\mathrm{c}$ & 6,95 & de & $11.203,7$ & d \\
\hline 7 & 4 & 5 & 0,11 & $\mathrm{~b}$ & 2,62 & e & $36.296,4$ & $\mathrm{~b}$ & 8 & 5 & 0,05 & $\mathrm{c}$ & 7,76 & de & $6.296,3$ & ef \\
\hline 8 & 5 & 4 & 0,09 & bc & 0,97 & $\mathrm{f}$ & $45.370,2$ & $\mathrm{a}$ & 6 & 5 & 0,05 & $\mathrm{c}$ & 4,70 & ef & $17.222,3$ & c \\
\hline 9 & 5 & - & 0,14 & $\mathrm{~b}$ & 8,67 & c & $28.857,6$ & $\mathrm{c}$ & 10 & - & 0,09 & b & 24,70 & b & $4.370,4$ & $\mathrm{fg}$ \\
\hline 10 & 4 & - & 0,13 & $\mathrm{~b}$ & 10,06 & b & $26.129,1$ & $\mathrm{c}$ & 8 & - & 0,07 & bc & 13,41 & $\mathrm{c}$ & $6.418,5$ & ef \\
\hline C.V(\%) & & & & 6,32 & & 6,57 & & 1,39 & & & &, 71 & & 11,24 & & 10,57 \\
\hline
\end{tabular}

${ }^{1}$ TRAT = Tratamentos: 1) Testemunha; 2$)$ Dimetomorfe + clorotalonil $(\mathrm{VSD}=10)+$ Metiram; 3$)$ Dimetomorfe + clorotalonil $(\mathrm{VSD}=12)+$ metiram; $)$ Dimetomorfe + mancozeb $(\mathrm{VSD}=8)$ + metiram; 5) Dimetomorfe + mancozeb $(\mathrm{VSD}=10)+$ metiram; 6) Piraclostrobina + metiram $(\mathrm{VSD}=8)+$ Metiram; 7) Piraclostrobina + metiram $(\mathrm{VSD}=10)+\mathrm{Metiram} ; 8) \mathrm{Uso}$ do calendário: Dimetomorfe + clorotalonil - Mancozeb - Metalaxil-M + Mancozeb - Clorotalonil alternados a cada sete dias, 9) Dimetomorfe + mancozeb (VSD = 8); 10) Dimetomorfe + clorotalonil (VSD

$=10$ ). O fungicida protetor metiram somente foi aplicado nas semanas em que não foi prevista nenhuma pulverização pelo sistema de previsão.

${ }_{13}^{12}$ Valor de severidade da doença atingido ao final do experimento.

${ }^{13}$ Média de 3 repetições:Médias seguidas por pelo menos uma mesma letra não diferem entre si pelo teste de Tukey a 5\% de probabilidade.

S: fungicida sistêmico $\quad \mathrm{P}$ : fungicida protetor $\quad-\quad$ = nenhuma pulverização realizada

Tabela 5. Número de pulverizações com fungicidas sistêmicos e protetores, área abaixo da curva de progresso da doença (AACPD), taxa de progresso da doença $(r)$ e produtividade influenciada pela requeima do tomateiro no experimento 3 .

\begin{tabular}{|c|c|c|c|c|c|c|c|c|}
\hline \multirow{4}{*}{$\begin{array}{c}\text { TRAT }^{1} \\
1\end{array}$} & \multicolumn{8}{|c|}{ EXPERIMENTO 3} \\
\hline & \multicolumn{2}{|c|}{$\mathrm{N}^{\circ}$ de pulverizações } & & \multirow{2}{*}{\multicolumn{2}{|c|}{ AACPD }} & \multirow{2}{*}{\multicolumn{2}{|c|}{$\begin{array}{c}\text { Produtividade } \\
\text { (Kg/ha) }\end{array}$}} \\
\hline & \multirow{2}{*}{$\frac{\text { Sistêmico }}{-}$} & \multirow{2}{*}{$\frac{\text { Protetor }}{-}$} & & & & & & \\
\hline & & & 0,25 & $r$ & $61,90^{13}$ & $\bar{a}$ & $2.175^{13}$ & $\mathrm{c}$ \\
\hline 2 & 4 & 5 & 0,05 & $\mathrm{c}$ & 1,58 & d & 59.145 & a \\
\hline 3 & 3 & 6 & 0,05 & $\mathrm{c}$ & 3,06 & d & 48.799 & $a b$ \\
\hline 4 & 4 & 5 & 0,08 & b & 6,09 & d & 34.864 & b \\
\hline 5 & 3 & 6 & 0,09 & b & 6,36 & d & 36.804 & b \\
\hline 6 & 5 & 3 & 0,08 & b & 6,17 & d & 33.746 & b \\
\hline 7 & 5 & 3 & 0,07 & $\mathrm{bc}$ & 5,05 & d & 38.744 & b \\
\hline 8 & 5 & 4 & 0,06 & $\mathrm{c}$ & 3,65 & d & 49.974 & $a b$ \\
\hline 9 & 4 & - & 0,10 & b & 25,91 & b & 7.231 & $\mathrm{c}$ \\
\hline 10 & 4 & - & 0,07 & $\mathrm{bc}$ & 17,04 & $\mathrm{c}$ & 12.581 & $\mathrm{c}$ \\
\hline C.V. $(\%)$ & & & & 18,15 & & 15,29 & & 11,97 \\
\hline VSD atin & ido $^{2}$ & & & & & & & \\
\hline
\end{tabular}

${ }^{11}$ TRAT = Tratamentos: 1$)$ testemunha; 2$)$ Dimetomorfe + clorotalonil $(\mathrm{VSD}=10)+$ metiram; 3$)$ Dimetomorfe + clorotalonil $(\mathrm{VSD}=12)+$ metiram; 4$) \mathrm{Metalaxil-M}+\mathrm{clorotalonil}(\mathrm{VSD}=10)$ + metiram; 5) Metalaxil-M + clorotalonil (VSD = 12) + metiram; 6) Dimetomorfe + mancozeb (VSD = 8) + metiram; 7) Piraclostrobina + metiram $(\mathrm{VSD}=8)+$ metiram; 8) uso do calendário:

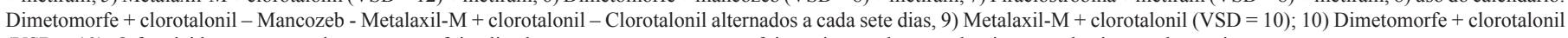
$(\mathrm{VSD}=10)$. O fungicida protetor metiram somente foi aplicado nas semanas em que não foi prevista nenhuma pulverização pelo sistema de previsão.

${ }^{12}$ Valor de severidade da doença atingido ao final do experimento.

${ }^{3}$ Média de 3 repetições:Medias seguidas por pelo menos uma mesma letra não diferem entre si pelo teste de Tukey a 5\% de probabilidade.

sistêmicos sem afetar a produtividade e a eficiência no controle da requeima. Nestes casos utilizou-se dimetomorfe + clorotalonil $(\mathrm{VSD}=10)+$ metiram $)$ e dimetomorfe + clorotalonil $(\mathrm{VSD}=12)+$ metiram, baseados no sistema de previsão de Wallin (1962). Resultados semelhantes foram obtidos por Costa et al. (5), os quais utilizaram o sistema de previsão de Wallin (1962) para o controle da requeima da batateira. Segundo esses autores, o uso do sistema de previsão com VSD-14 permitiu reduzir o número de pulverizações com fungicida sistêmico sem que houvesse perda significativa na produção. Costa et al. (4), em experimento realizado com tomate de mesa, também relatou redução no número de pulverizações com fungicida sistêmico empregando-se VSD de 1416 em 1998, e VSDs de 11-13 e 14-16 em 1999, obtendo a mesma eficiência do calendário de pulverização semanal no controle da requeima. A redução do número de pulverizações com fungicida sistêmico obtida com o uso do sistema de previsão proporciona menor custo de produção, pois os fungicidas sistêmicos são mais caros, além disso, há redução de resíduos nos frutos e menor agressão ao meio ambiente.

A redução do número de pulverizações com o uso do sistema de previsão pode não ser regra geral, isto porque sob condições climáticas muito favoráveis ao desenvolvimento da doença pode não haver redução 
do número de pulverizações, podendo em determinados casos até haver aumento no número de pulverizações (16). No experimento 2, isto foi observado, onde as condições climáticas foram muito favoráveis à requeima e o tratamento 2 (dimetomorfe + clorotalonil $(\mathrm{VSD}=10)+$ metiram) baseado no sistema de previsão de Wallin (1962) foi o mais eficiente no controle da requeima e apresentou duas pulverizações a mais com fungicida sistêmico, comparado ao sistema de calendário de pulverizações semanais. Porém este mesmo tratamento apresentou produtividade superior comparado aos demais tratamentos.

$\mathrm{O}$ uso apenas de fungicida sistêmico de acordo com o sistema de previsão não foi eficiente no controle da requeima (tratamentos $9 \mathrm{e}$ 10) mesmo utilizando-se o menor VSD. O tratamento 2 onde foi utilizado fungicida protetor em alternância com o fungicida sistêmico dimetomorfe, apresentou controle mais eficiente, independente das condições climáticas. Esse resultado mostra a importância da alternância da aplicação do fungicida protetor na semana em que o sistema de previsão não indicar nenhuma pulverização de fungicida sistêmico. Este esquema além de aumentar a eficiência no controle da requeima, previne o surgimento de populações resistentes de $P$. infestans.

O modelo de Previsão de Wallin (1962) é baseado na umidade relativa do ar ( $\geq 90 \%$ ) e temperatura média diária. Neste trabalho, o modelo foi modificado para a duração do molhamento foliar e temperatura média durante o período e foram mantidos os mesmos períodos de duração, originalmente designados para umidade relativa. Segundo Jones et al. (10) essa modificação é devido à umidade relativa não ser uma variável adequada para se prever molhamento foliar, podendo resultar em previsões incorretas em função da tendência à formação inicial de orvalho, com umidade relativa mais baixa no inverno do que no verão, além da perda de energia pela folha depende da umidade absoluta do ar e da formação de nuvens. Costa et al. (5) observou períodos de molhamento foliar com a umidade relativa do ar em torno de $80 \%$. No modelo original baseado na umidade relativa considerava-se como molhamento foliar apenas quando a umidade relativa do ar fosse $\geq 90 \%$, com isso o sistema de previsão poderia superestimar ou subestimar o número de pulverizações.

Neste trabalho o sistema de calendário de pulverização semanal utilizado apresentou número de pulverizações inferior ao observado em produtores, principalmente aqueles que utilizam cultivares suscetíveis, os quais realizam de duas a três pulverizações por semana quando as condições climáticas são favoráveis à requeima.

De acordo com o resultado das análises realizadas, tanto a taxa de progresso da doença $(r)$ quanto a área abaixo da curva de progresso da doença (AACPD) discriminaram os tratamentos do experimento 1. No experimento 2 a AACPD discriminou melhor os tratamentos comparada à taxa de progresso da doença, e no experimento 3 a $r$ discriminou melhor os tratamentos comparada à AACPD. Nazareno et al. (18) alerta quanto a necessidade de atenção quando se avalia apenas a AACPD ou apenas a taxa de progresso da doença, isto porque curvas de progresso com mesmo valor de AACPD podem apresentar diferenças quanto ao tempo inicial, severidade final e à taxa de progresso da doença, também taxas de progresso da doença semelhantes podem apresentar diferenças quanto a AACPD.

Para a utilização do sistema de previsão de Wallin em diferentes localidades, os valores de VSD devem ser ajustados para cada cultivar e condições climáticas locais. Para as condições climáticas de Viçosa-MG, nos três experimentos realizados, a utilização de dimetomorfe + clorotalonil $(\mathrm{VSD}=10)$ + metiram foi o tratamento mais eficiente no controle da requeima, independente da época de cultivo.

\section{REFERÊNCIAS BIBLIOGRÁFICAS}

1. Bergamin Filho, A.; Amorim, L. Sistemas de previsão e avisos. In: Bergamin Filho, A., Kimati, H., Amorim, L. (Ed.) Manual de fitopatologia. São Paulo: Agronômica Ceres, 1995.v.1, p.602-626.

2. Camargo Filho, W.P.; Mazzei, A.R. Mercado mundial de tomate e o mercosul. Informações Econômicas. São Paulo, v.27, n.10, p.25-38, 1997.

3. Campbell, C. L.; Madden, L. V. Introduction to plant disease epidemiology. New York: John Wiley, 1990. 532 p.

4. Costa, R.V.; Zambolim, L.; Vale, F.X.R.; Mizubuti, E.S.G. Prediction system for tomato late blight. Summa Phytopathologica, Botucatu, v.31, n.1, p.14-20, 2005.

5. Costa, R.V.; Zambolim, L.; Vale, F.X.R.; Mizubuti, E.S.G. Previsão da requeima da batateira. Fitopatologia Brasileira, Brasília, v.27, n.4, p.349-354, 2002

6. Fohner, G.R.; Fry, W.E.; White, G.B. Computer simulation raises question about timing protecting fungicide application frequency according to potato late blight forecast. Phytopathology, St. Paul, v.74, n.10, p.1145-1147, 1984.

7. Hyre, R.A. Progress in forecasting late blight of potato and tomato. Plant Disease Reporter, Illinois, v. 38, n. 4, p. $245-$ 253,1954 .

8. James, W.C. A manual of assessement keys for plant diseases. Ottawa. Departament of Agriculture . 1971. Publ. n ${ }^{\circ} 1458$

9. Jesus Junior, W.C. de; Pozza, E.A.; Vale, F.X.R. ; Mora-Aguilera, G. Análise Temporal de Epidemia. In: Vale, F.X.R.; Jesus Junior, W.C. de; Zambolim, L. (Ed.) Epidemiologia aplicada ao manejo de doenças de plantas. Belo Horizonte, Perfil Editora: 2004. p.125-192.

10. Jones, A.L. Role of wet periods in predicting foliar disease. In: Leonards, K.J.; Fry, W.E. (Ed). Plant disease epidemiology: population dynamics and management. New York: MacMillan, 1986. v.1, p.87-89.

11. Jones, J.B.; Stall, R.E.; Zitter,T.A. Compedium of tomato deseases. St Paul: APS, 1993. 73p.

12. Kurozawa, C.; Pavan, M.A. Doenças do Tomateiro (Lycopersicon esculentum). In: Kimati, H., Amorim, L., Rezende, J. A.M., Bergamim Filho, A.; Camargo, L.E.A.(Ed.). Manual de fitopatologia. São Paulo: Agronômica Ceres, 2005. v.2, p.607-626.

13. Lopes, C.A.; Santos, J.R.M. Doenças do tomateiro. Brasília: EMPRAPA/CNPH, 1994. 67 p.

14. Maffia, L.A., Martins, M.C.P., Matsuoka, K. Doenças do tomateiro. Informe Agropecuário, Belo Horizonte, v. 6, n. 66, p.42 - 60, 1980.

15. Makishima, N. Colheita, classificação, embalagem e comercialização. Informe Agropecuário, Belo Horizonte, v.6,n.66, p.6163,1980

16. Mizubuti, E.S.G. Sistemas de Previsão de Doenças de Plantas: Uma Ferramenta Útil? In: Zambolim, L. (Ed.). Manejo integrado de doenças e pragas. Visconde do Rio Branco: Suprema Gráfica e Editora, 1999.v.1, p.42-46.

17. Mizubuti, E.S.G.; Fry,W.E. Temperature effects on developmental stages of isolates from three clonal lineages of Phytophthora infestans. Phytopathology, St. Paul, v.88, n.8, p.837843,1998

18. Nazareno, N.R.X.; Scotti, C.A.; Mafioletti, R.L.; Boschetto, N. Controle da requeima da batata através do monitoramento das variáveis climáticas. Fitopatologia Brasileira, v. 24, n. 2, p. 170 - 174, 1999.

19. Pitiblado, R.E. Development of a weather - timed fungicide spray program for field tomatoes. Canadian Journal of Plant Pathology, Guelph, v. 10, n.1, p. 371, 1988.

20. Royle, D.J.; Shaw, M.W. The costs and benefits of disease forecasting in farming practice. In: Clifford, B. C.; Lester, E. (Eds.). Control of plant disease: costs and benefits. Blackwell: Oxford, 1988. v.1, p. $231-246$.

21. Silva, J.B.C.; Giordano, L.B. Produção mundial e nacional. In: Silva, J.B.C.; Giordano, L.B (Ed.). Tomate para processamento industrial. Brasília: EMBRAPA Hortaliças, 2000. v.1, p.8-11. 
22. Stevenson, W.R. An integrated program for managing potato late blight. Plant Disease, St. Paul, v.67, n.9, p.1047-1048, 1983.

23. Thurston, H.D.; Schultz, O. Late blight. In: Hooker W.J, (Ed.). Compendium of potato diseases. St. Paul: APS, 1981. p.40-42.

24. Torres, J.C.; Ventura, J.A. AACPD: um programa para calcular a área e o volume abaixo da curva de progresso da doença. Fitopatologia Brasileira, Brasília, v. 16, supl., p. 52, 1991.

25. Vale, F.X.R.; Zambolim, L.; Chaves, G.M.; Correia, L.G. Avaliação fitossanitária da cultura do tomateiro em regiões produtoras de Minas Gerais e Espírito Santo. Fitopatologia Brasileira, Brasília, v. 17, n. 2, p. 211, 1992.

26. Vale, F.X.R.; Zambolim, L.; Paul, P.A., Costa, H. Doenças causadas por fungos em tomate. In: Zambolim, L.; Vale, F.X.R.; Costa, H.(Ed).
Controle de doenças de plantas- Hortaliças.Visconde do Rio Branco: Suprema Gráfica e Editora, 2000. v.2, p.173-207.

27. Wallin, J.R. Summary of recent progress in predicting late blight epidemics in United States and Canada. American Potato Journal, Orono, v. 39, n.3, p.306 - 312, 1962.

28. Zambolim, L.; Vale, F.X.R.; Costa, H. Doenças causadas por fungos em batata. In: Zambolim, L.; Vale, F.X.R.; Costa, H. (Ed.). Controle de doenças de plantas: Hortaliças. Visconde do Rio Branco: Suprema Gráfica e Editora, 2000. v.1, cap.5, p.173-207.

29. Zambolim, L.; Vale, F.X.R.; Cruz Filho, J.; Chaves, G.M. Emprego da Calda Viçosa na cultura do tomateiro (L. esculentum) para o controle de doenças da parte aérea. Informe técnico 66. Viçosa: Universidade Federal de Viçosa, 1990. 7 p. 